\title{
Los proyectos de vinculación una estrategia de mejora en la gestión contable del sector microempresarial
}

\author{
The projects link an improvement strategy in the accounting management of the microenterprise sector
}

Os projetos vinculando uma estratégia de melhoria na gestão contábil do setor de microempresa

\section{Kenia Lizzeth Carchi Arias \\ kcarchi@umet.edu.ec \\ https://orcid.org/0000-0003-4023-4015}

Universidad Metropolitana, Machala-Ecuador

Martín Andrés Romero Lalangui

martin.romero.l@est.umet.edu.ec

https://orcid.org/0000-0002-7465-618X

Universidad Metropolitana, Machala-Ecuador

\section{Fernando Xavier Juca Maldonado \\ fuca@umet.edu.ec \\ https://orcid.org/0000-0001-7430-2157}

Universidad Metropolitana, Machala-Ecuador

Betzy Lizbeth Orellana Arévalo

betzy.orellana@est.umet.edu.ec

https://orcid.org/0000-0002-9050-1989

Universidad Metropolitana, Machala-Ecuador

Artículo recibido en junio 2021, revisado en julio 2021, arbitrado en agosto 2021 y publicado en septiembre 2021

\section{RESUMEN}

El presente artículo tuvo como objetivo describir el nivel de desarrollo de potencialidades en la gestión contable y tributaria de los microempresarios como resultado de las capacitaciones en línea, las mismas que fueron disertadas por estudiantes involucrados en el proyecto de vinculación de la carrera de Contabilidad y Auditoría de la Universidad Metropolitana. Desde la integración de la profesionalización alumnodocente se fortalece la investigación, y el compromiso social frente a los actores externos. La metodología fue descriptivalongitudinal, porque muestra el nivel de mejora en el aprendizaje adquirido por los participantes; concluyendo que el $81 \%$ de los aprobados alcanzaron un nivel de recuperación del $23 \%$, en relación con el promedio inicial. Así como también la valoración del test post hoc expone que el mayor rendimiento obtenido de los seis cursos; tributación básica y Excel intermedio tiene promedios que están en un rango de 90 a 100 puntos, considerándolos de mayor puntuación.

Palabras clave: Capacitación en línea; TIC; Contabilidad; Microempresas

\section{ABSTRACT}

The present article aims to describe the level of development of potentialities in the accounting and tax management of microentrepreneurs as a result of online training, which were given by students involved in the project to link the Accounting and Auditing career. from the Metropolitan University. From the integration of the student-teacher professionalization, research and social commitment are strengthened against external actors. The methodology was descriptive-longitudinal, because it shows the level of improvement in learning acquired by the participants; concluding that $81 \%$ of those approved reached a recovery level of $23 \%$, in relation to the initial average. As well as the evaluation of the post hoc test shows that the highest performance obtained from the six courses; Basic taxation and intermediate Excel have averages that are in a range of 90 to 100 points, considering them to be the highest score.

Key words: Online training; ICT; Accounting; Micro-businesses

\section{RESUMO}

O objetivo deste artigo é descrever o nível de desenvolvimento de potencialidades na gestão contábil e tributária de microempresários em decorrência dos treinamentos online, ministrados por alunos envolvidos no projeto de vinculação à carreira de Contabilidade e Auditoria. Universidade. A partir da integração da profissionalização alunoprofessor, a pesquisa e o compromisso social se fortalecem contra os atores externos. A metodologia foi descritivalongitudinal, pois mostra o grau de melhoria na aprendizagem adquirida pelos participantes; concluindo que $81 \%$ dos aprovados atingiram um índice de recuperação de $23 \%$, em relação à média inicial. Bem como a avaliação do teste post hoc mostra que o melhor desempenho obtido nas seis disciplinas; A tributação básica e o Excel intermediário têm médias que variam de 90 a 100 pontos, considerando-se a pontuação mais alta.

Palavras-chave: Treinamento on-line; TIC; Contabilidade; Microempresas 


\section{INTRODUCCIÓN}

Uno de los factores determinantes que agobia al sector microempresarial es su nivel de educación, por cuanto el $70 \%$ solo tienen instrucción primaria y secundaria altos, conduciéndolos a trabajar de forma empírica y limitando su desarrollo empresarial, mismo que no les permite competir en mercados internacionales (Texis, Ramírez, y Aguilar, 2016). Es por ello que desde la academia se educa a los estudiantes, proporcionándoles bases teóricas, conduciéndolos a la praxis para afianzar conocimientos, mediante la preparación y actualización constante de conocimientos en las normas contables, tributaria vigente; de modo que sean capaces de responder oportunamente a las necesidades que demanda la sociedad, a través de los proyectos de vinculación.

Los estudiantes de quinto, sexto y séptimo semestre de la carrera de Contabilidad y Auditoría, se involucran en actividades de vinculación desde la asignatura de Práctica Principal Integradora I, II y III, cuyos resultados de aprendizaje buscan proponer soluciones a problemas de la profesión enfocados a la realidad nacional e internacional; diagnosticar, diseñar e implantar sistemas contables y financieros para cualquiera de las formas de organización económica con la aplicación e integración de las técnicas más avanzadas del ejercicio de la profesión, $y$; el uso eficiente de herramientas ofimáticas que apoyen el procesamiento automatizado de la información económica, dando cumplimiento a lo establecido en la malla curricular de la carrera.

Un proyecto de vinculación se define como un proceso sustantivo e interrelacionado, en el que intervienen docentes y estudiantes en cooperación con una entidad beneficiaria, que aporte con alternativas de soluciones viables ante las necesidades detectadas en un grupo vulnerable (Navarrete Pita et. al., 2020). En consecuencia, este artículo está fundamentado en lo establecido por la Constitución del Ecuador (2008) artículo N³43 que expone, "El sistema nacional de educación tendrá como finalidad el desarrollo de capacidades y potencialidades individuales y colectivas de la población, que posibiliten el aprendizaje, y la generación y utilización de conocimientos, técnicas, saberes, artes y cultura" (p. 160). En el mismo cuerpo legal en el artículo $\mathrm{N}^{\circ} 347$, inciso 8 establece como responsabilidad del Estado "Incorporar las tecnologías de la información y comunicación en el proceso educativo y propiciar el enlace de la enseñanza con las actividades productivas o sociales" (Constitución del Ecuador, 2008, p. 161).

Dentro de este contexto legal, la vinculación con la sociedad compromete a los involucrados a actuar con responsabilidad social en la transformación social, difusión y devolución de conocimientos académicos (Galvão et.al. 2019). El proyecto de vinculación titulado "Desarrollo de potencialidades en el área de contabilidad y tributación con el uso de las TIC's', tiene como fin desarrollar las habilidades y destrezas de los microempresarios mediante capacitaciones gratuitas, en modalidad virtual, con la adaptación de herramientas informáticas a los procedimientos contables que manejan los microempresarios de la Provincia de El Oro, Ecuador; mismo que está amparado en convenio con el Colegio de Contadores de El Oro, Ecuador.

El presente trabajo de investigación tuvo como objetivo principal, describir el nivel de conocimiento alcanzado de los participantes que aprobaron los seis cursos dictados bajo la modalidad en línea, con el uso de la Plataforma virtual Eva, desde junio 2020 hasta mayo 2021. Así como también se muestra un análisis de comparación de medias de los promedios finales obtenidos después de la evaluación de los seis cursos asistidos, dando a conocer los cursos que alcanzaron el mejor rendimiento de aprendizaje 
que garantiza la apropiación de conocimientos prácticos para el buen desempeño de los alumnos aprobados, en las diferentes áreas u ocupaciones a las que se dedican.

\section{Revisión literaria}

La variable de estudio que describe este trabajo es la vinculación con la sociedad, y cómo este contribuye al aprendizaje de los microempresarios a partir de las capacitaciones en línea en temas de contabilidad y afines al área.

Barreno Salinas et al., (2018) definen a la vinculación con la sociedad como "el vínculo entre la formación académica de los estudiantes desde el punto de vista teórico-práctico y su inclusión en el mercado laboral" (p. 41). Su objetivo se ve evidenciado en querer entablar nuevas relaciones tales como la ciencia, tecnología, sociedad y el desarrollo, con la estrategia de elaborar proyectos de carácter social y fomentar el trabajo en conjunto con las comunidades.

Llevar a cabo proyectos de vinculación con la sociedad en las instituciones de educación superior es relevante, ya que proporciona experiencia acerca del proceso de construcción del conocimiento, apreciación de perspectivas múltiples, y favorece la participación en el proceso de aprendizaje, insertando los conocimientos en la experiencia y sumergirse más en la realidad social (Rivas Tello et al., 2017). Con la realización de los proyectos de vinculación se involucra al estudiante a hacer investigación científica, a perfeccionar su perfil en el área contable y tributaria, que sea capaz de atender a la sociedad con conocimientos sólidos y eficaces a través de las capacitaciones gratuitas.

Salomón et al. (2012) señala que "la capacitación se convierte en un instrumento indispensable para fortalecer los conocimientos y habilidades de los investigadores en campos que no conocieron en su proceso formativo" (p. 5). Sin duda, desarrollar una investigación de calidad requiere de tiempo y búsqueda de información dictados desde los diferentes talleres, seminarios y cursos, en los que se evidenciará como un proceso estimulante, responsable y comprometido con el desarrollo de la ciencia y la satisfacción de las necesidades nacionales, ya que con ello se permite dar respuesta a los problemas sociales que encaran en nuestro entorno.

En el mismo contexto también se presentan los tipos de modalidades en las que se pueden ofertar las capacitaciones, tales como las presenciales y virtuales. La diferencia más importante entre la educación presencial y virtual es el medio de instrucción utilizado, que se deriva de la flexibilidad del tiempo, y optimización de costos; que se traduce en que las dos modalidades buscan llegar al mismo resultado, optando por diferentes caminos (Sangrá Morer, 2017).

Por la situación actual de pandemia, el tipo de modalidad más utilizado es la virtual, ya sea por ser estrictamente necesaria o por la rutina diaria que se vive en el campo laboral (Vásquez Ponce, Juleisy, José, y Chara Plúa, 2020). Entre los beneficios que ofrece este tipo de modalidad se enlistan las siguientes: flexibilidad, variedad de programas y cursos, comunicación asincrónica, fomenta la responsabilidad y la autonomía, reducción de costos, equilibrio entre estudio y trabajo.

La modalidad virtual utiliza medios de enseñanza sincrónico y asincrónico. El método sincrónico es caracterizado por la interacción con los asistentes mediante encuentros virtuales o videoconferencias, que facilite la comunicación directa con el docente, asegurando que el grado de aprendizaje sea el esperado; así como también ayuda a disipar dudas sobre la temática abordada, y recibir direccionamiento en el desarrollo de las actividades. 
Por otra parte, el método asincrónico favorece a reforzar el conocimiento mediante el feedback que el estudiante debe realizar para el cumplimiento de sus tareas, teniendo la facilidad de organizar el tiempo a su conveniencia y la oportunidad de interactuar mediante el uso de plataformas virtuales (Flores Ferrer y Bravo, 2012).

Como parte del proceso de la ejecución de capacitaciones en línea existen entornos virtuales, conocido como el espacio de aprendizaje que le permite al docente gestionar la distribución de información y recursos didácticos necesarios, de acuerdo a los resultados de aprendizaje entablados a su disciplina. Es fundamental mencionar que la adaptación de las TIC's a los procesos educativos conlleva la utilización de metodologías de trabajo que aseguren el intercambio de información entre estudiante y docente mediante el entorno virtual. Flores Ferrer y Bravo (2012) expone la importancia que tiene la aplicación de metodologías en el proceso de enseñanza, el cual sugiere la metodología PACIE, cuya sigla significan: (P) Presencia, (A) Alcance, (C) Capacitación, (I) Interacción y (E) E-learning; la misma que aporta al aprendizaje colaborativo y desarrollo de competencias de forma autónoma.

Es fundamental reconocer que la oferta de un curso no debe de considerarse como un gasto o despilfarro de dinero, sino más bien como una inversión a largo plazo, en que la organización o persona natural que lo patrocine va a obtener beneficios y frutos importantes. En este aspecto Boudet (2017) manifiesta que:

la capacitación es un proceso constante que busca la eficiencia y con ella alcanzar niveles elevados de productividad" y que además "tiene como fin primordial motivar al capital humano para que se conciba en un ambiente de trabajo atractivo que les permita trabajar en conjunto y en el que pueda desarrollar su capacidad creativa y productiva (p. 5).

Entre las oportunidades que brindan las capacitaciones en línea han sido instruir a los microempresarios; que pese al escaso conocimiento que poseen de la gestión administrativa y financiera, muestran una aptitud competente con una amplia posibilidad de sobrevivencia en el mercado local. De hecho, es sorprendente ver cómo algunas microempresas siguen en el mercado, careciendo del uso de técnicas y estrategias de gestión comercial para desempeñar sus actividades. Sin embargo, Sumba Bustamante y Santistevan Villacreses (2018) exponen en su investigación, que "los microempresarios iniciaron la actividad comercial como una oportunidad de obtener ingresos, sin conocimiento formal de actividad empresarial, y que los resultados obtenidos reflejan el bajo nivel de educación, considerando necesario que se los capacite" (p. 325).

A raíz de la pandemia del Covid-19 surgen las adaptaciones tecnológicas a los programas de capacitación propuestos por el proyecto de vinculación antes mencionado. En este caso, el tipo de formación sobre las cuales se sustentaron las actividades es la modalidad virtual, utilizando la metodología PACIE.

\section{MÉTODO}

La investigación propuesta fue de tipo descriptiva, pues se detallaron las características de la gestión contable que requieren los microempresarios, como unidad de estudio, en relación a la falta de herramientas contables, financieras e informáticas que posee, y su escaza capacidad de adaptación a los cambios surgidos por las competencias y nuevas tecnologías. Así 
mismo, el estudio se complementó con un estudio longitudinal realizado a las calificaciones obtenidas antes, durante y al final de las actividades de formación, que determina el nivel de apropiación de conocimientos de los participantes en los diferentes cursos en los que interactuaron.

Para detectar las necesidades de capacitación que oferta el proyecto que hace mención este trabajo, se aplicó una encuesta a 420 microempresarios no obligados a llevar contabilidad que infieren en la población de 82.564 contribuyentes con RUC y RISE con su actividad económica activa de la provincia de El Oro, Ecuador (Base de datos proporcionada por el Servicio de Rentas Internas hasta diciembre 2019).

También se realizó un análisis documental para fundamentar teóricamente las variables de estudio, es decir conocer la importancia que tienen los proyectos de vinculación en la formación académica del estudiante y su aporte como alternativa viable en la transformación social; adicionalmente, se caracterizó la capacitación virtual, y medios de enseñanza recomendadas para su ejecución. En el proyecto se implementaron herramientas informáticas como Google Forms para evaluar los conocimientos adquiridos por los participantes, así como el Excel para procesar la información e interpretar gráficamente los resultados obtenidos.
Para la determinación de la muestra se utilizó la fórmula de cálculo del tamaño de la muestra, partiendo de la población de 82.564 habitantes que representan la provincia de El Oro, Ecuador, con un nivel de confianza del 95\%, que significa que los resultados probablemente cubrirán las expectativas del investigador. De cierto modo también se consideró un nivel de probabilidad del 50\% de que ocurra el evento que se estudia.

Se aplicó la siguiente fórmula:

$$
n=\frac{N \times Z_{a}^{2} \times p \times q}{d^{2} \times(N-1)+Z_{a}^{2} \times p \times q}
$$

El tipo de muestreo utilizado fue probabilístico, aplicando un método aleatorio simple, debido a que toda la población objetivo tiene la misma posibilidad de ser seleccionada. Para el levantamiento de la información se consideró a los cantones: Machala, Pasaje, Huaquillas, Santa Rosa, El Guabo; puesto que la sumatoria de los 5 cantones pertenecientes a Ecuador representan un valor significativo del $82 \%$ del total de la población que corrobora con la necesidad de estudio del proyecto, así como lo muestra la Tabla 1.

Tabla 1. Distribución del tamaño de la muestra.

\begin{tabular}{lccc}
\hline \multicolumn{1}{c}{ Cantón } & Nro. & Pi & ni \\
\hline Machala & 38.177 & 0,565 & 246 \\
Pasaje & 8.942 & 0,132 & 53 \\
Huaquillas & 7.603 & 0,112 & 45 \\
Santa Rosa & 7.270 & 0,108 & 43 \\
El Guabo & 5.617 & 0,083 & 33 \\
Total & $\mathbf{6 7 . 6 0 9}$ & & $\mathbf{4 2 0}$ \\
\hline
\end{tabular}

Fuente: Base de Datos SRI. 
Con la identificación de las necesidades del microempresario, fue necesaria la preparación de una estructura coherente de capacitaciones que garantice suplir las necesidades y vacíos de información del mismo, a través del uso de herramientas informáticas adecuadas, apoyadas con la realización de diversas clases online.

Con los datos obtenidos al final de las capacitaciones, fue posible realizar un análisis comparativo entre los promedios finales de cada uno de los cursos creados por la universidad, profundizando en el mismos a través del uso del software estadístico SPSS, con el cual fue posible la formulación de una comparación de medias entre los promedios finales de cada uno de los contenidos vistos.

\section{RESULTADOS Y DISCUSIÓN}

\section{Detección de necesidades de capacitación a los microempresarios}

Como resultado de las encuestas aplicadas, el $80 \%$ manifestaron el interés en aprender temas en relación a la contabilidad básica y uso de TIC's, en cuanto al desconocimiento que tienen en el manejo de las páginas institucionales, al uso de herramientas informáticas aplicadas a los negocios y dificultades en la determinación de su utilidad económica; así como también, el 94\% manifiesto interés en capacitarse sobre temas tributarios, dado a los cambios constantes en las reformas tributarias y poco conocimiento en el cálculo de IVA, aplicación de retenciones y otros conceptos tributarios para el pago de impuestos, tal como muestra el Gráfico 1.

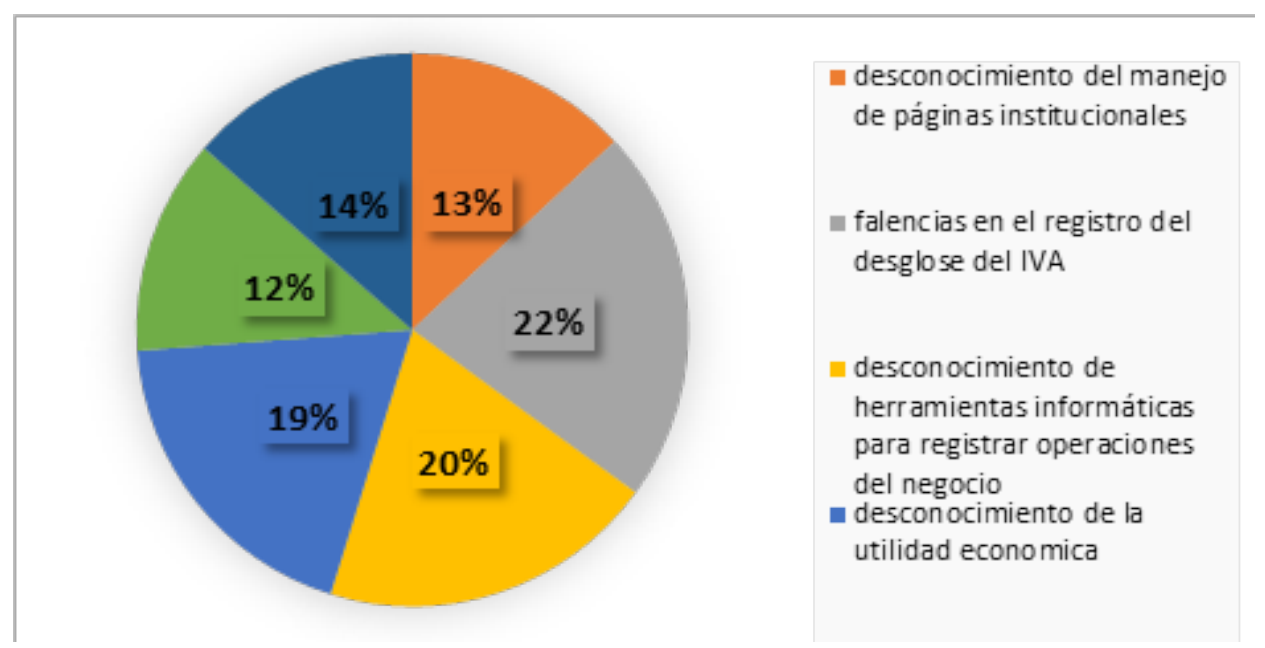

Gráfico 1. Actividades que los microempresarios desarrollan con mayores dificultades. (Fuente: Encuesta aplicada a las 420 microempresas). 
Por otra parte, el 75\% de los encuestados en los dos últimos años no han recibido capacitaciones en temas contables y tributarios o de otras índoles, información que justifica el medio empírico de administrar las finanzas de sus negocios, poniendo en riesgo sus inversiones; sin embargo, el 35\% si han recibido asesoramiento en el área de contabilidad y tributación. En general el 92\% manifestó que si está interesado en asistir a las capacitaciones gratuitas que oferta el proyecto.

\section{Planificación, organización y ejecución de las capacitaciones}

A partir de los resultados obtenidos de la encuesta, se procedió a elaborar programas de capacitación de seis cursos distintos, partiendo del análisis de las necesidades que demandan los beneficiarios; en el mismo documento se establecen objetivos, resultados de aprendizaje por cada tema, junto con el cronograma respectivo para la organización y ejecución de las actividades de capacitación. De esta forma, el primer período inicio con tres cursos de nivel básico, conforme a la participación y aprobación de los microempresarios se proponen 3 cursos adicionales de nivel intermedio, que fortalezcan el conocimiento adquirido en las capacitaciones iniciales.

En la Tabla 2 se muestran los contenidos mínimos de los cursos de nivel básico, los cuáles se abordaron entre 4 a 5 fines de semana, con una duración de 40 a 48 horas trabajadas.

Tabla 2. Contenidos mínimos de cursos de nivel básico.

\begin{tabular}{|c|c|c|}
\hline Cursos & Contenidos & Horas \\
\hline \multirow[t]{4}{*}{$\begin{array}{l}\text { Contabilidad Básica } \\
\text { para Microempresas }\end{array}$} & $\begin{array}{l}\text { Tema 1. Fundamentos básicos de la Contabilidad y marco conceptual Sección } 3 \text { y } 4 \\
\text { de NIIF para PYMES }\end{array}$ & 8 \\
\hline & Tema 2. Partes de la cuenta contable y ecuación contable & 8 \\
\hline & Tema 3: Aplicación del Proceso contable & 16 \\
\hline & Tema 4: Elaboración del estado de situación financiera y estado de resultados & 16 \\
\hline \multirow{4}{*}{$\begin{array}{l}\text { Tributación Básica para } \\
\text { Microempresas }\end{array}$} & Tema 1. Obligaciones y deberes formales & 8 \\
\hline & Tema 2. RUC Y RISE & 8 \\
\hline & Tema 3. Comprobantes de venta y de retención & 12 \\
\hline & Tema 4. Declaración del Impuesto al Valor Agregado & 12 \\
\hline \multirow{4}{*}{$\begin{array}{l}\text { Excel básico para } \\
\text { microempresas }\end{array}$} & Tema 1: Tipos de Datos & 8 \\
\hline & Tema 2: Fórmulas de cálculo & 8 \\
\hline & Tema 3: Funciones & 12 \\
\hline & Tema 4: Gráficos estadísticos & 12 \\
\hline
\end{tabular}

Fuente: Programas de capacitación elaborados por el proyecto de vinculación "Desarrollo de las potencialidades en el área de contabilidad, tributación con el uso de las TIC's" 
En la Tabla 3 se muestran los contenidos mínimos de los cursos de nivel intermedio, los cuáles se abordaron entre 3 a 4 fines de semana, con una duración de 40 horas trabajadas.

Tabla 3. Contenidos mínimos de cursos de nivel intermedio.

\begin{tabular}{|c|c|c|}
\hline Cursos & Contenidos & Horas \\
\hline \multirow{6}{*}{$\begin{array}{l}\text { Contabilidad } \\
\text { Intermedia aplicando } \\
\text { la sección } 13 \text { Inventario } \\
\text { NIIF para PYMES }\end{array}$} & Tema 1. Importancia de la aplicación de normas contables y tributarias en la & 8 \\
\hline & contabilidad & \\
\hline & Tema 2. Tratamiento del Inventario en la actividad comercial sección 13 NIIF para & 8 \\
\hline & Pymes & \\
\hline & Tema 3: Aplicación del Proceso contable & 12 \\
\hline & Tema 4: Elaboración y análisis de los estados Financieros & 12 \\
\hline \multirow[t]{4}{*}{ Tributación Intermedia } & Tema 1. Normas Tributarias & 8 \\
\hline & Tema 2. Gastos personales & 8 \\
\hline & Tema 3. Análisis de los formularios 104 y 102 & 12 \\
\hline & Tema 4. Cálculo y presentación del impuesto de la renta global & 12 \\
\hline \multirow{4}{*}{$\begin{array}{l}\text { Excel Intermedio para } \\
\text { microempresas }\end{array}$} & Tema 1: Tabla de datos & 8 \\
\hline & Tema 2: Ordenar, filtrar y sub-totalizar datos & 8 \\
\hline & Tema 3: Tablas dinámicas & 12 \\
\hline & Tema 4: Gráficos dinámicos & 12 \\
\hline
\end{tabular}

Fuente: Programas de capacitación elaborados por el proyecto de vinculación "Desarrollo de las potencialidades en el área de contabilidad, tributación con el uso de las TIC's"

Para la ejecución de las capacitaciones fue primordial organizar a los grupos de trabajo para perfeccionar el perfil estudiantil mediante capacitaciones de aprobación de los temas específicos que responden a los programas de contenidos que muestra la Tabla 2 y 3 , permitiendo afianzar los conocimientos desde la academia; investigación para el desarrollo de los recursos didácticos y las guías de estudio dirigidos a los microempresarios. Para ello se involucraron a 30 estudiantes de la carrera de Contabilidad y Auditoría del quinto, sexto y séptimo semestre. Así mismo, se dictó un curso de Estrategias de Enseñanzas con una duración de 20 horas, para que el estudiante identifique las técnicas de enseñanzas oportunas y sean capaces de incorporarlas en el material y ejercicios prácticos del curso propuesto; también se los capacitó en el uso de la plataforma virtual como facilitadores, lo cual les permitirá preparar el Entorno Virtual de Aprendizaje con el contenido a utilizar en los distintos cursos.

Una vez planificado y organizado los cursos se procedió a promocionarlos a través de las redes sociales, correos, WhatsApp, obteniendo un total de 1.355 personas inscritos, de los cuales el 51\% tienen un nivel de educación primaria y secundaria. No obstante, en la Tabla 4 se observa que el $48.63 \%$ que son 659 microempresarios asistieron con 
regularidad a los encuentros virtuales y accedieron al Aula Virtual a consultar los recursos. De modo que 537 aprueban los cursos, obteniendo una nota mínima de 70 y máxima de 100/100 puntos. De los 537 aprobados el 26\% corresponden al curso de contabilidad básica, el $8 \%$ en contabilidad intermedia, el 20\% en Excel básico, el 9\% en Excel intermedio, el 22\% en tributación básica y el 16\% en tributación intermedia.
También se analizaron los motivos de la no aprobación del 19\%, concluyendo que las causas son externas a la logística y al manejo de las capacitaciones, puesto que se evidenció la falta de entrega de actividades y realización de los cuestionarios propuestos en la plataforma virtual, perjudicando la nota del promedio final.

Tabla 4. Participantes inscritos y aprobados.

\begin{tabular}{lccc}
\hline \multicolumn{1}{c}{ Nombre de los cursos } & Número Inscritos & Asistencia regular & Número de aprobados \\
\hline Contabilidad Básica & 382 & 163 & 137 \\
Contabilidad Intermedia & 104 & 65 & 43 \\
Excel básico & 304 & 116 & 106 \\
Excel Intermedio & 136 & 75 & 50 \\
Tributación Básica & 275 & 151 & 117 \\
Tributación Intermedia & 154 & 89 & 84 \\
Total & $\mathbf{1 . 3 5 5}$ & $\mathbf{6 5 9}$ & $\mathbf{5 3 7}$ \\
\hline
\end{tabular}

Fuente: Datos proporcionados por el formulario de inscripción a los cursos y actas de calificaciones.

Después del proceso de evaluación se determinó el nivel de conocimiento alcanzado, para ello se realizó un análisis comparativo entre la nota inicial que se obtuvo de la evaluación de diagnóstico, y la nota final promedio de cada evaluación aplicada.

En la Tabla 5 se observa que, de los 537 participantes aprobados, el $26 \%$ que corresponden a contabilidad básica, obtienen un promedio final de 85/100 puntos, alcanzado un $33 \%$ en mejora de sus habilidades y competencias en el registro contable de las operaciones diarias de la actividad económica, permitiéndoles direccionar los procesos contables oportunamente en la toma de decisiones empresariales. Así mismo, el 22\% de aprobados de tributación básica obtiene una nota promedio final de 92/100 puntos, alcanzado un nivel de aprendizaje del 19\%, sobre las normas tributarias básicas para el buen manejo de las transacciones diarias de un negocio, que sustenten los ingresos y gastos que exige la ley tributaria.

Por otra parte, el 20\% de los aprobados en Excel básico alcanzan el 32\% de desarrollo de habilidades técnicas en el ingreso y procesamientos de datos, que contribuya en la creación de gráficos e interpretación de información contable de los negocios, obteniendo una nota final promedio de 88/100 puntos; así como también, el 8\% de aprobados en contabilidad intermedia, alcanzan el $23 \%$ de apropiación de conocimiento teórico y competencias en el reconocimiento y medición del inventario, según la NIIF para PYMES, con una nota final promedio de 85/100 puntos; también del $16 \%$ de aprobados en tributación intermedia, obtienen un nota final promedio de 86/100 puntos, 
alcanzado el 10\% de adquisición y actualización de conocimientos relacionados a la reforma tributaria sobre la liquidación, declaración y pago del impuesto a la renta de las personas naturales no obligadas a llevar contabilidad, que evite el pago de multas e intereses que representa flujo de efectivo negativo en la empresa.
Por último, el 9\% de los aprobados en Excel intermedio, alcanzan el $19 \%$ de desarrollo de potencialidades en el manejo de tablas de datos y dinámicas para el análisis de información e interpretación de resultados de la gestión administrativa, contable o financiera en una empresa.

Tabla 5. Nivel de conocimiento alcanzado de los microempresarios aprobados.

\begin{tabular}{lcccc}
\hline \multicolumn{1}{c}{ Nombre de los cursos } & $\begin{array}{c}\text { Número de } \\
\text { aprobados }\end{array}$ & $\begin{array}{c}\text { Promedio } \\
\text { inicial }\end{array}$ & $\begin{array}{c}\text { Promedio } \\
\text { final }\end{array}$ & $\begin{array}{c}\text { Nivel de } \\
\text { conocimientos } \\
\text { alcanzados }\end{array}$ \\
\hline Contabilidad Básica & 137 & 64 & 85 & $33 \%$ \\
Tributación Básica & 117 & 77 & 92 & $19 \%$ \\
Excel básico & 106 & 67 & 88 & $32 \%$ \\
Contabilidad Intermedia & 43 & 69 & 85 & $23 \%$ \\
Tributación Intermedia & 84 & 78 & 86 & $10 \%$ \\
Excel Intermedio & 50 & 77 & 92 & $19 \%$ \\
\hline
\end{tabular}

Fuente: Datos proporcionados por los cuestionarios aplicados como medio de evaluación de los cursos.

\section{Nivelesdesatisfaccióndelosmicroempresarios aprobados}

El nivel de satisfacción de los participantes que asistieron con regularidad a las clases virtuales, es del $92,28 \%$ como promedio de los resultados obtenidos por cada curso, manifestaron estar muy satisfechos con el conocimiento impartido, y con la logística para desarrollar las capacitaciones y sobre todo el $89,5 \%$ consideran estar muy de acuerdo con el dominio y la forma de enseñar de los estudiantes facilitadores; cumpliendo con el 92\% de las expectativas que tuvieron los asistentes sobre el nivel de aprendizaje alcanzado, resultados favorables que influyeron en que el $100 \%$ de los partícipes recomienden los cursos a otras personas.

\section{Análisis de comparación de medias en relación a las notas finales de los aprobados}

Para la comparación de medias, se consideró como variable dependiente los promedios finales de los cursos y como factor el tipo de curso recibido (ya sea contabilidad, tributación o Excel básico/ intermedio para microempresarios); se hizo uso del software estadístico SPSS, en el cual, una vez ingresados los datos, se procedió a cotejar las medias a través del ANOVA de un factor, de lo cual se obtuvieron los siguientes resultados.

Como lo muestra la Tabla 6, primero se obtuvieron las estadísticas descriptivas por los promedios finales de las capacitaciones. En la columna "N" con el número de aprobados de cada 
curso, en "Media" estando la calificación promedio final de cada curso; en "desviación típica" la medida en que las calificaciones obtenidas por los estudiantes están dispersos respecto a la media; en "Error Típico" que determina las oscilaciones de la calificación promedio de cada curso; se obtienen también los límites superiores e inferiores de los intervalos de confianza para la media al 95\%; y por último las calificaciones mínimas y máximas obtenidas por los aprobados de cada curso.

Tabla 6. Análisis de comparación de media sobre el promedio final obtenido por el microempresario.

\begin{tabular}{|c|c|c|c|c|c|c|c|c|}
\hline & \multirow[t]{2}{*}{$\mathbf{N}$} & \multirow[t]{2}{*}{ Media } & \multirow[t]{2}{*}{$\begin{array}{c}\text { Desviación } \\
\text { típica }\end{array}$} & \multirow[t]{2}{*}{$\begin{array}{l}\text { Error } \\
\text { típico }\end{array}$} & \multicolumn{2}{|c|}{$\begin{array}{c}\text { Intervalo de } \\
\text { confianza para la } \\
\text { media al } 95 \%\end{array}$} & \multirow[t]{2}{*}{ Mínimo } & \multirow[t]{2}{*}{ Máximo } \\
\hline & & & & & $\begin{array}{l}\text { Límite } \\
\text { inferior }\end{array}$ & $\begin{array}{l}\text { Límite } \\
\text { superior }\end{array}$ & & \\
\hline Contabilidad Básica & 135 & 85,5540 & 10,22716 & 88021 & 83,8131 & 87,2949 & 64,00 & 100,00 \\
\hline Tributación Básica & 117 & 91,8986 & 7,83958 & ,72477 & 90,4631 & 93,3341 & 60,00 & 100,00 \\
\hline Excel Básico & 108 & 85,8735 & 10,83835 & 1,04292 & 83,8060 & 87,9410 & 70,00 & 100,00 \\
\hline Contabilidad Intermedia & 43 & 86,9070 & 8,30036 & 1,26579 & 84,3525 & 89,4614 & 70,00 & 100,00 \\
\hline Tributación Intermedia & 84 & 86,6394 & 8,71915 & ,95134 & 84,7472 & 88,5316 & 70,00 & 100,00 \\
\hline Excel Intermedio & 50 & 92,4950 & 6,58645 & ,93146 & 90,6232 & 94,3668 & 73,00 & 100,00 \\
\hline Total & 537 & 87,9250 & 9,59361 & 41399 & 87,1118 & 88,7383 & 60,00 & 100,00 \\
\hline
\end{tabular}

Fuente: Acta de calificaciones, analizado mediante el SPSS.

A continuación, la Tabla 7 muestra los resultados de la comparación de medias a partir del ANOVA de un factor; el cuadro presenta la suma de cuadrados, los grados de libertad, la media cuadrada y el F estadístico; sin embargo, el valor que permitirá determinar si existe o no una diferencia significativa entre la media de los promedios finales de cada curso será el valor de "Sig." (o el nivel de significancia), que en este caso, al poseer un valor inferior al $5 \%$ es posible afirmar que existe una diferencia significativa en la media de al menos dos de los cursos analizados.

Tabla 7. Análisis de ANOVA Promedio final obtenido por el microempresario.

\begin{tabular}{|c|c|c|c|c|c|c|}
\hline & $\begin{array}{l}\text { Suma de } \\
\text { cuadrados }\end{array}$ & gl & & Media cuadrática & $\mathbf{F}$ & Sig. \\
\hline Inter-grupos & 4288,490 & & 5 & 857,698 & 10,111 & ,0000000029 \\
\hline Intra-grupos & 45043,511 & & 531 & 84,828 & & \\
\hline Total & 49332,000 & & 536 & & & \\
\hline
\end{tabular}

Fuente: Acta de calificaciones, analizado mediante el SPSS. 
Con la finalidad de conocer cuál o cuáles medias hacen la diferencia, se realizó un test post hoc, utilizando un supuesto de varianzas iguales tipo "Tukey-b" a un nivel de significancia igual a 0,05. En la Tabla 8 se observa que las medias de contabilidad básica, Excel básico, tributación intermedia y contabilidad intermedia no mantienen diferencias significativas entre sí; no obstante, esto cambia al ser comparadas con las medias de los grupos de tributación básica y Excel intermedio, debido a que estos dos cursos no presentan diferencias significativas entre sí, por los promedios que están dentro del rango de 90 a 100. Lo que significa que en ambos cursos se obtuvieron mejores rendimientos o apropiación de conocimientos por parte de los participantes.

Tabla 8. Rendimiento alcanzado por el microempresario, Tukey B.

\begin{tabular}{llcc}
\hline \multicolumn{1}{c}{$\begin{array}{c}\text { Capacitación a la que asistió el } \\
\text { microempresario }\end{array}$} & N & \multicolumn{1}{c}{ Subconjunto para alfa $=\mathbf{. 0 5}$} \\
\hline Contabilidad Básica & 135 & 85,5540 & $\mathbf{2}$ \\
Excel Básico & 108 & 85,8735 & \\
Tributación Intermedia & 84 & 86,6394 & 91,8986 \\
Contabilidad Intermedia & 43 & 86,9070 & 92,4950 \\
Tributación Básica & 117 & & \\
Excel Intermedio & 50 & & \\
\hline
\end{tabular}

Se muestran las medias para los grupos en los subconjuntos homogéneos.

a. Usa el tamaño muestral de la media armónica $=74,651$.

b. Los tamaños de los grupos no son iguales. Se utilizará la media armónica de los tamaños de los grupos. Los niveles de error de tipo I no están garantizados.

\section{Discusión}

De acuerdo al diario El Universo (2020) el 56\% de los ecuatorianos tienen acceso a las tecnologías, como el internet; pero el $10,7 \%$ de la población entre 15 y 49 años padecen de analfabetismo digital, cuya brecha podría cubrirse a través de las capacitaciones en línea (Boudet, 2017), ya que como menciona Valencia Flores, et. al (2017) la capacitación constante permitirá que alcancen los niveles de preparación necesarios para mejorar sus habilidades digitales, que sirva de apoya a las competencias laborales y personales.

Por ello, Vásquez Ponce et al. (2020) manifiesta que la alternativa más viable en la que los estudiantes y facilitadores puedan optar si desean comunicarse entre sí, es la enseñanza online, la cual compromete a todos los involucrados a participar con responsabilidad a través de la comunicación sincrónica y asincrónica. Es por ello que Boudet et al. (2017) manifiestan que la aplicación de los procesos de capacitación dirigidos a los microempresarios va siempre asociados a procesos de cambio $y$, por tanto, dirigidos a preparar a los dueños del negocio para lograr que pueda proteger sus intereses y orientarse al logro de sus objetivos. Valencia Flores et al. (2017) consideran que los microempresarios ecuatorianos requieren capacitación en temas de los principales conceptos de teorías y prácticas contables, así como de técnicas y herramientas que les permitirá mejorar sus organizaciones y manejar 
de manera eficiente y responsable sus negocios, con la ayuda de las capacitaciones podrán apropiarse de estos temas y hacer que sus negocios respondan oportunamente a los cambios del entorno y que genere una rentabilidad económica deseada.

\section{CONCLUSIONES}

Del estudio realizado, se concluye que el $2 \%$ del totaldela población objetivo tuvieron la oportunidad de beneficiarse de las diferentes capacitaciones ofertados por el proyecto de vinculación, generando un impacto favorable del $81 \%$ de aprobados en relación a 659 microempresarios que asistieron regularmente, alcanzando un rendimiento promedio de 88/100 puntos. Se considera como aceptable y exitoso el número de beneficiados del sector microempresarial, y haber contribuido de esta forma en su mejora en el área contable al optimizar y facilitar la toma de decisiones oportunas y mejorar su competitividad en el campo laboral.

Los datos estadísticos de la Tabla 8, muestran que los cursos con mayor rendimiento fueron los de tributación básica y Excel intermedio, alcanzado notas de 92/100 respectivamente. En general, esto significa que el $29 \%$ de los microempresarios aprobados respondieron acertadamente a los resultados de aprendizaje en cuanto al reconocimiento de deberes formales y obligaciones tributarias, con el plus de haber potenciado sus habilidades digitales en el uso de Excel.

Por otra parte, la percepción de los participantes ha sido muy satisfactoria en cuanto a su participación de las actividades y capacitaciones, pues consideran que los estudiantes facilitadores demostraron preparación y dominio de los temas impartidos, así como también un buen manejo en la organización y gestión logística, el entorno virtual de aprendizaje utilizado, la metodología aplicada $y$ otros elementos que influyeron para cubrir el
$100 \%$ de las expectativas de los microempresarios participantes, dando como resultado indirecto su total recomendación de las capacitaciones impartidas.

Finalmente, es recomendable que el microempresario se capacite de forma continua, y promueva el desarrollo integral del personal, propiciando $\mathrm{y}$ fortaleciendo el conocimiento $\mathrm{y}$ habilidades necesarias para el mejorar el desempeño de sus actividades comerciales y organizacionales. Resultados que se esperan presentar en una segunda etapa del proyecto que mide el impacto de la capacitación y como tributan los conocimientos teóricos y prácticos en el desempeño de sus negocios.

REFERENCIAS

Barreno Salinas, M., Barreno Salinas, Z., y Olmedo Valencia, A. C. (2018). La educación superior y su vinculación con la sociedad: referentes esenciales para un cambio. Revista Universidad y Sociedad, 10(3),40-45. http://scielo.sld.cu/ scielo.php?script $=$ sci_arttextypid $=$ S22 18 $36202018000300040 \mathrm{ylng}=$ esytlng=es

Boudet, L. V. (2017). Evaluación del impacto de la capacitación en directivos de instalaciones turísticas. Ciencia, docencia y tecnología, 28, 130-149. http://www.scielo.org.ar/pdf/cdyt/ n54/n54a05.pdf

Constitución del Ecuador 218. (2008). Obtenido de https://www.asambleanacional.gob.ec/sites/ default/files/documents/old/constitucion_de_ bolsillo.pdf

El Universo. (marzo de 2020). Educar en línea, un reto por el limitado acceso a Internet en Ecuador. Obtenido de https://www.eluniverso.com/ noticias/2020/03/22/nota/7789911/educacionlinea-ecuador-internet-clases/

Flores Ferrer, K. M., y Bravo, M. d. (2012). Metodología PACIE en los ambientes virtuales de aprendizaje para el logro de un aprendizaje colaborativo. Revista electrónica Diálogos Educativos, 12. Obtenido de file://C:/Users/ 
Windows/Downloads/Dialnet-Metodologia PACIEEnLosAmbientesVirtualesDeAprendi za-4156135.pdf

Galvão, A., Mendes, L., Marques, C., y Mascarenhas, C. (de 2019). Factors influencing students' corporate social responsibility orientation in higher education. Revista de producción más limpia, 215, 290-304. doi:https://doi. org/10.1016/j.jclepro.2019.01.059

Navarrete Pita, Y., Zambrano Intriago, G. Z., Alcivar Molina, S. A., y Rodríguez Fiallos, J. L. (2020). Incidencia de los proyectos de vinculación con la sociedad y su contribución a la educación de los estudiantes. Revista Estudios del Desarrollo Social: Cuba y América Latina, 8(2). doi:http://scielo.sld.cu/scielo.php?script $=$ sci_ arttextypid=S2308-01322020000200006

Rivas Tello, A. C., Ángel Poma, M. d., y López López, M. d. (2017). Efectos del modelo de vinculación con la sociedad de la Facultad de comunicación social de la Universidad Central del Ecuador, desde la perspectiva de entornos de aprendizaje. Revista de Ciencias de Seguridad y Defensa 2, 2(1). http://geo1.espe.edu.ec/wp-content/ uploads/2017/10/art5.pdf

Salomón, L., Medina, E., y Amador, J. (2012). La promoción de la investigación científica a través de la capacitación metodológica y técnica. Revista Ciencia y Tecnología (10), 3-14. doi:https://doi.org/10.5377/rct. v0i10.1059
Sangrá Morer, A. (2017). Educación a distancia, educación presencial y usos de la tecnología: una tríada para el progreso educativo. Revista Electrónica de TecnologíaEducativa (15). doi:https://doi.org/10.21556/ edutec.2002.15.541

Sumba Bustamente, R., y Santistevan Villacreses, K. L. (2018). Las microempresas y la necesidad de fortalecimiento: reflexiones de la zona sur de Manabí. Universidad y Sociedad, 10(5). http://scielo.sld.cu/scielo.php? script $=$ sci_ arttextypid=S2218-36202018000500323

Texis, M., Ramírez, M., y Aguilar, J. G. (2016). Microempresas de base social y sus posibilidades de supervivencia. ELSEVIER- Contaduría y Administración, Vol. 61.(3), 551-567. doi: 10.1016/j.cya.2015.04.001

Valencia Flores, C., Isaac Godínez, C. L., y Brito Vallina, M. L. (2017). Programa de capacitación a microempresarios del centro histórico de Quito. Universidad y Sociedad, 9(4), 70-74. https://rus.ucf.edu.cu/index.php/rus/article/ view/666/778

Vásquez Ponce, G. O., Juleisy, I. F., José, F. M., y Chara Plúa, E. J. (2020). Educación virtual en tiempos del covid-19 desde la perspectiva socioeconómica de los estudiantes de la Universidad Estatal del Sur de. Polo del Conocimiento, 5(10), 798-823. doi:10.23857/ pc.v5i10.1852 